\title{
HIRA and Daxx Constitute Two Independent Histone H3.3-Containing Predeposition Complexes
}

\author{
S.J. Elsaesser AND C.D. Allis \\ Laboratory of Chromatin Biology and Epigenetics, The Rockefeller University, \\ New York, New York 10065 \\ Correspondence: alliscd@rockefeller.edu
}

\begin{abstract}
Histone H3.3 is a universal replacement histone in metazoans that has been implicated in diverse processes ranging from gene activation to heterochromatin silencing. Here, we show that, before deposition, H3.3 exists in two biochemically distinct complexes, associated with either Daxx or HIRA, Ubinuclein-1, and Cabin-1. Although the HIRA complex is evolutionarily conserved in yeast, Daxx is a novel histone chaperone unique to metazoans. Deletion of HIRA in mouse embryonic stem cells impairs the HIRA complex integrity but does not abolish Daxx association with H3.3/H4. Similarly, HIRA interacts with H3.3/H4 in the absence of Daxx. We hypothesize that these two H3.3 chaperone systems provide separate pools of H3/H4 units for incorporation at distinct sites within the genome. We provide evidence that the association of histone H3.3 with distinct assembly systems allows it to acquire unique posttranslational modifications before deposition that might affect its role after incorporation into chromatin.
\end{abstract}

Histone octamers package the eukaryotic genome, not only compacting the DNA, but also encoding and propagating a sophisticated additional layer of epigenetic information. Despite the inherent physical stability of the nucleosome particle, the resulting chromatin structure must be highly dynamic to allow access to the DNA-encoded information. $\mathrm{H} 3 / \mathrm{H} 4$ and $\mathrm{H} 2 \mathrm{~A} / \mathrm{H} 2 \mathrm{~B}$ units are deposited and evicted from nucleosomes by different histone chaperones and ATP-dependent chromatin remodelers. Histone chaperones thereby serve two major functions: They (1) buffer the pool of nonchromatin-associated histones in predeposition complexes and (2) facilitate favorable histone-DNA interactions during the stepwise assembly of the histone octamer-DNA complex from H3/H4 and H2A/H2B units. Consequently, mutants of histone chaperones in Saccharomyces cerevisiae cannot form proper chromatin structure (Sharp et al. 2002; Adkins and Tyler 2004; Andrews et al. 2010).

Metazoans have evolved histone variants to meet the diverse needs for establishing and maintaining complex epigenetic information. Histone H3.3 is a minor variant of the canonical S-phase histones H3.1 and H3.2 in metazoans. However, its role as a replacement histone outside of S phase is conserved to lower eukaryotes, and accumulating evidence suggests that, by virtue of a unique amino acid motif, H3.3 engages in several variant-specific chromatin assembly pathways. Whereas the amino acid sequence of $\mathrm{H} 3.3$ is $>95 \%$ identical to $\mathrm{H} 3.1 / 2$, the small AAIG motif in the core histone fold (Fig. 1) allows targeting of the variant to specific genomic loci by a replication-independent mechanism (Ahmad and Henikoff 2002; Goldberg et al. 2010). The existence of identical H3 variants throughout the metazoan lineage underscores their importance as carriers of epigenetic information in somatic cells and the germline (for review, see Campos and Reinberg 2010; Elsaesser et al. 2010).
Reading out the subtle differences between H3.3 and $\mathrm{H} 3.1 / 2$ poses an intricate structural problem to variantspecific chaperones. Hence, a number of recent studies have aimed to elucidate which proteins confer specificity in chromatin assembly. By purifying H3.1- and H3.3-associated complexes from HeLa nuclear extracts, the replication-independent histone deposition factor HIRA was found to associate specifically with H3.3 (Tagami et al. 2004). Using the same approach, we and other investigators identified a different polypeptide, the Fas death-domain-associated protein Daxx, as an H3.3-specific binding protein (Drané et al. 2010; Goldberg et al. 2010; Lewis et al. 2010). We found that Daxx specifically interacts with the AAIG motif of H3.3 in vitro and is a bona fide H3.3-specific histone chaperone (Lewis et al. 2010). The ATP-dependent chromatin remodeler and $\alpha$-thalassemia X-linked mental retardation protein (ATRX) formed a complex with Daxx-H3.3/H4 that was essential for H3.3 chromatin assembly in vivo (Goldberg et al. 2010; Lewis et al. 2010).

Although we were able to detect HIRA in H3.3-associated fractions, this association was unstable following exposure to the more stringent biochemical conditions used to identify Daxx. This raised the question of whether HIRA and its associated proteins Ubinuclein-1 and Cabin1 were recruited indirectly, potentially via a secondary interaction with Daxx or another as yet unidentified protein. We addressed this issue by biochemically fractionating H3.3-containing subcomplexes from HeLa cells and purifying $\mathrm{H} 3$.3-associated proteins from mouse embryonic stem cells (ESCs) devoid of HIRA, Daxx, or ATRX. We find that the HIRA/Ubinuclein-1/Cabin-1 and ATRX/ Daxx complexes are two separate biochemical entities that independently associate with $\mathrm{H} 3.3$. We have previously shown that HIRA is responsible for incorporation of H3.3 at genic regions, whereas ATRX is required for telomeric 


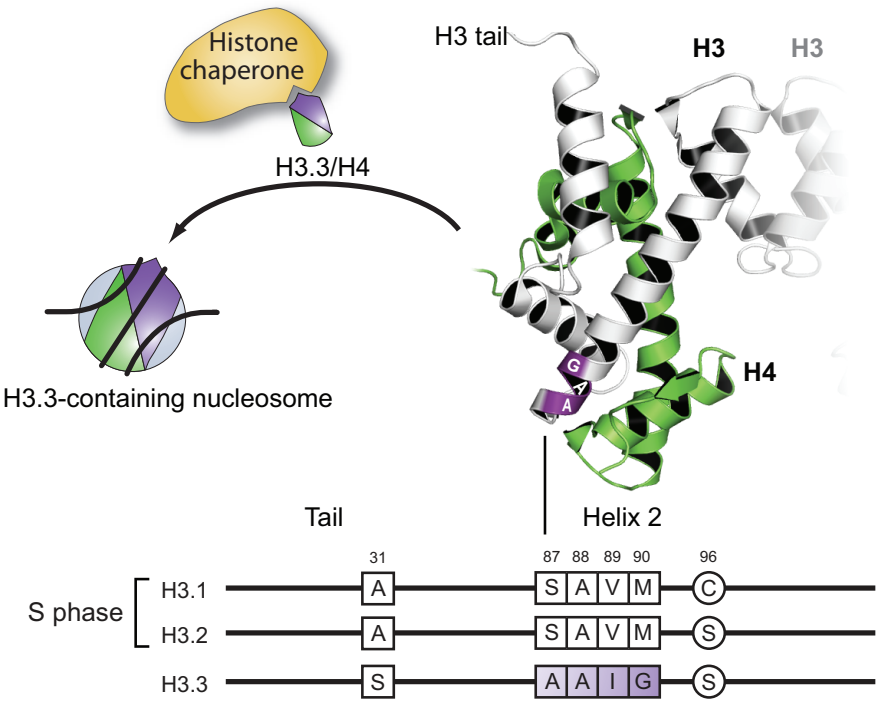

Figure 1. A highly conserved AAIG motif in the core histone fold of metazoan H3.3. Schematic of noncentromeric histone $\mathrm{H} 3$ variants in mammals. H3.1 and H3.2 are commonly referred to as canonical or S-phase histone H3. Variable amino acids are shown with residue number. Three unique residues of H3.3 form the AAIG motif at the base of helix 2 of the histone $\mathrm{H} 3$ core (purple; note that isoleucine is hidden in the helical depiction). The molecular structure of a representative $\mathrm{H} 3 / \mathrm{H} 4$ dimer ([H3] white, [H4] green) has been adapted from the nucleosome structure (PDB:1kx5). The H3:H3 interface of the $\mathrm{H} 3 / \mathrm{H} 4$ tetramer is indicated. deposition of H3.3 (Goldberg et al. 2010). Therefore, HIRA and Daxx/ATRX constitute two separate histone H3.3-deposition machineries that act on distinct genomic loci.

\section{MATERIALS AND METHODS}

Nuclear extracts were prepared as described in Lewis et al. (2010) from HeLa cells stably expressing FLAG-HAtagged H3.1 or H3 (Tagami et al. 2004) or mouse ESCs (Goldberg et al. 2010). Immunoprecipitations were performed in 20-mM HEPES $\cdot \mathrm{HCl}$ (pH 7.5), $150 \mathrm{~mm} \mathrm{NaCl}$, $10 \%$ glycerol, 0.2 mM EDTA, 2 mM $\mathrm{MgCl}_{2}$ in the presence of DNase and RNase, washed five times with wash buffer (20 mM HEPES• $\mathrm{HCl}$ [pH 7.5], $250 \mathrm{~mm} \mathrm{NaCl}, 10 \%$ glycerol, 0.2 mM EDTA, 0.01\% NP-40 [Nonidet P-40]) and eluted with either FLAG peptide or 1\% SDS. Eluted material was separated by SDS polyacrylamide gel electrophoresis (SDS PAGE) and analyzed by western blotting with the following antibodies: $\alpha$-FLAG-HRP (SigmaAldrich), $\alpha$-HA-HRP A190-107P (Bethyl), $\alpha$-HIRA WC119 (Peter Scambler), $\alpha$-p150 sc-10772 (Santa Cruz), $\alpha$-Daxx sc-7152 (Santa Cruz), $\alpha$-p60 NB500-212 (Novus Biologicals), $\alpha$-Cabin-1 ab3349 (Abcam), $\alpha$-ATRX H-300 (Santa Cruz), $\alpha$-ASF1a/b 4A1/3 (Santa Cruz), $\alpha-H 3$ K9Ac, No. 07-352 (Millipore), $\alpha$-acetyl-H4, No. 06-598 (Millipore), and $\alpha$-H4K5Ac, No. 06-759 (Millipore).

\section{BIOCHEMICAL FRACTIONATION OF H3.3-CONTAINING COMPLEXES}

Previous studies used double-affinity purification of FLAG-HA-tagged H3.3 (e-H3.3) from nuclear extracts to isolate H3.3-associated factors (Tagami et al. 2004; Drané et al. 2010; Lewis et al. 2010). However, this strategy did not allow us to identify putative subcomplexes that independently coimmunoprecipitate with H3.3. Thus, we developed an alternative biochemical approach to fractionate
H3.3-containing complexes from nuclear extracts based on their affinity to a negatively charged heparin matrix.

We noted that e-H3.3 eluted over a broad range of salt concentrations from immobilized heparin (Fig. 2A). Whereas HIRA and CAF-1 p150 complexes elute with the bulk of nuclear proteins at medium salt, Daxx only dissociated at higher salt concentrations from the heparin column (Fig. 2A). Therefore, this approach provided us with a means to separate a distinct subpopulation of e-H3.3 and associated proteins. We combined fractions containing almost exclusively HIRA/CAF-1 p150 or Daxx and immunoprecipitated e-H3.3 (Fig. 2B). HIRA, but not CAF-1 p150, copurified with H3.3. Because Daxx was absent from the immunoprecipitated material, we conclude that HIRA associates specifically with H3.3, independent of Daxx. As a control, we heparin-fractionated e-H3.1 nuclear extracts (data not shown) and performed immunoprecipitation from the corresponding HIRA/CAF-1 p 150 fractions; this e-H3.1 immunoprecipitation yielded CAF1 p150 (Fig. 2B). Daxx coimmunoprecipitated with H3.3 from the higher salt fractions in the absence of HIRA (Fig. 2B). Thus, we were able to isolate nonoverlapping H3.3 subcomplexes containing either HIRA or Daxx from heparin-fractionated nuclear extract.

In addition to HIRA, we found Cabin-1 and Ubinuclein1 associated with the first pool of e-H3.3/H4 (Fig. 2C). HIRA, Cabin-1, and Ubinuclein-1 have previously been found to form a complex in the presence and absence of H3.3/H4 (Tagami et al. 2004; Drané et al. 2010). Iterative alignment searches identified Cabin-1 and Ubinuclein-1 as mammalian homologs of the yeast proteins Hir3 and Hcp2p, respectively, suggesting a HIRA complex analogous to the yeast Hir complex. The control H3.1 immunoprecipitation yielded all three subunits of the CAF-1 complex: p150, p60, and RbAp46/48.

Daxx coprecipitated with H3.3 from the higher salt fractions without apparent stoichiometric partners (Fig. $2 \mathrm{C}, \mathrm{D})$, in agreement with our previous observation that 
A
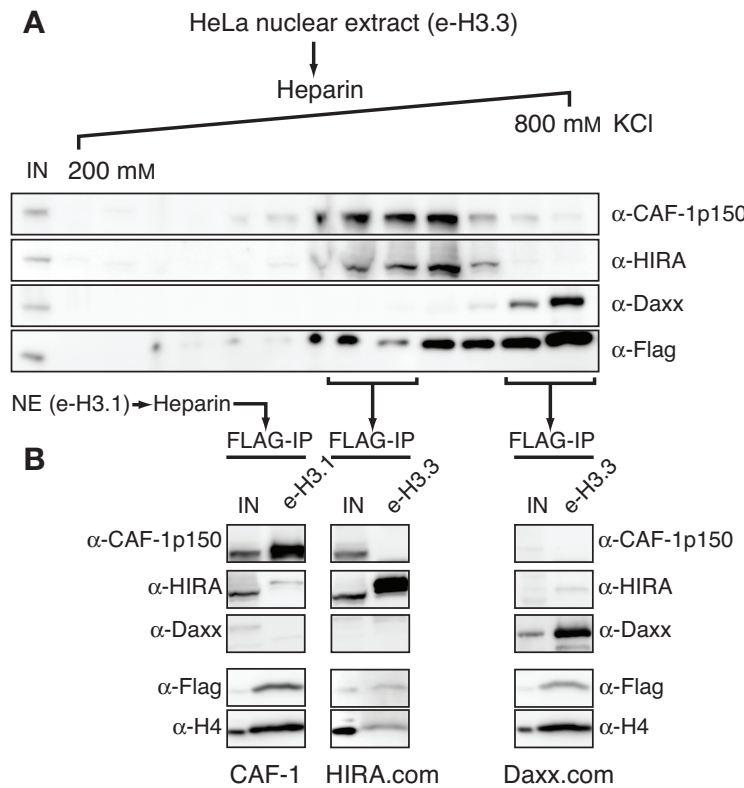

C

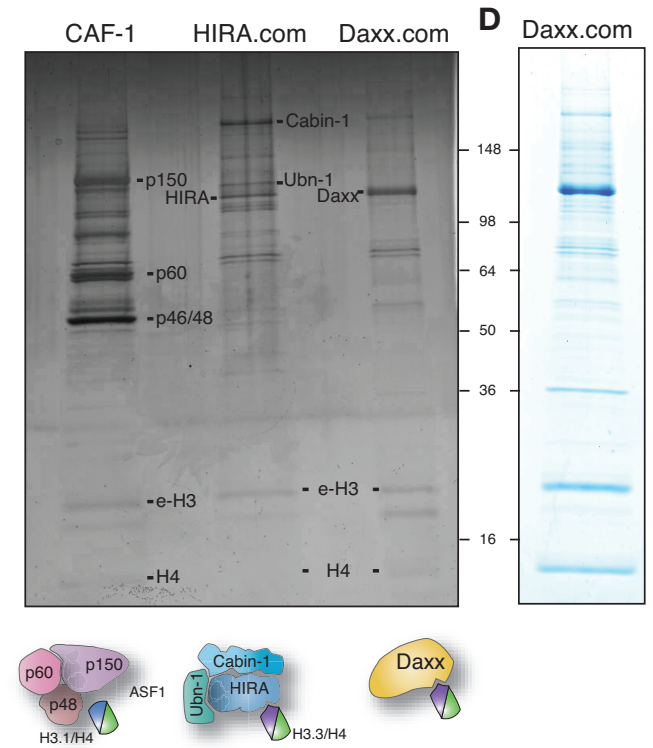

Figure 2. HIRA and Daxx constitute two biochemically distinct H3.3-containing subcomplexes. (A) Biochemical fractionation of nuclear $\mathrm{H} 3.3$ complexes: $200-800-\mathrm{mM} \mathrm{KCl}$ fractions from a heparin column were probed for e-H3.3, HIRA, CAF-1, and Daxx by western blotting. ( $B$ ) Affinity purification of e-H3.3 and e-H3.1 complexes. e-H3.3/H3.1 complexes were immunoprecipitated from indicated pools and analyzed by western blotting. Immunoprecipitation from the medium-salt e-H3.3 and e- $\mathrm{H} 3.1$ fractions yielded predominantly HIRA and CAF-1, respectively; Daxx coprecipitated with high-salt e-H3.3. (C) Silver staining of eluted CAF-1, HIRA, and Daxx complexes from above. Indicated bands were all identified by mass spectrometry and/or verified by western blotting. (D) Coomassie stain of the Daxx complex estimates protein abundance.

the direct interaction of Daxx with H3.3/H4 is not dependent on other proteins such as ATRX (Lewis et al. 2010). In conclusion, we find that the known H3.3-associated proteins fractionate into two biochemically distinct subcomplexes constituted by HIRA and Daxx.

\section{GENETIC DISSECTION OF H3.3-CONTAINING COMPLEXES}

Next, we assessed how the deletion of either HIRA or Daxx histone chaperones affects H3.3 and its associated complexes in respective knockout mouse ESC lines. We used a zinc-finger-based genome editing strategy to specifically tag one allele of the endogenous $\mathrm{H} 3.3 \mathrm{~B}$ gene in wildtype W9.5, HIRA $^{--}$, Daxx ${ }^{--}$, and ATRX ${ }^{--}$ESCs with a carboxy-terminal HA tag (H3.3-HA) (Goldberg et al. 2010), assuring endogenous expression levels of the tagged histone. We found elevated levels of H3.3-HA in nuclear extracts of HIRA ${ }^{--}$ESCs, as previously reported (Meshorer et al. 2006), whereas the soluble pool of H3.3-HA was reduced in Daxx ${ }^{-1}$ ESCs (Fig. 3, left). We speculate that Daxx buffers a considerable pool of $\mathrm{H} 3.3$ in the nucleus by forming a stable complex with $\mathrm{H} 3 / \mathrm{H} 4$ units. Overall, $\mathrm{H} 3$ and $\mathrm{H} 4$ levels (accounting for the untagged H3.3 as well as H3.1/2) in the nucleoplasm were unchanged, indicating that reduced levels of H3.3 might be compensated by an increase in soluble H3.1/2. Levels of chromatin-bound H3.3-HA were comparable in all cell lines (Fig. 3, bottom).

As previously reported, ATRX levels were reduced in Daxx $^{-1}$ cells (Lewis et al. 2010). Unexpectedly, Cabin-1 was depleted in $\mathrm{HIRA}^{--}$nuclear extracts (Fig. 3, left panels) and whole-cell lysates (data not shown). Cabin-1 mRNA levels are not perturbed in $\mathrm{HIRA}^{-/}$cells (Goldberg et al. 2010), suggesting that the Cabin-1 protein might be unstable and degraded in the absence of HIRA. Therefore, Cabin-1 likely contributes directly to the function of the HIRA complex, as already shown for Ubinuclein-1 (Banumathy et al. 2009).

\section{HIRA and Daxx Complexes IndependentlyBind H3.3}

Based on our biochemical fractionation of H3.3-containing complexes, we hypothesized that association of HIRA and Daxx with H3.3 occurs independently. We tested this by immunoprecipitating H3.3-HA from the above ESC nuclear extract. Indeed, HIRA copurified with H3.3-HA in Daxx $^{-/}$cells and vice versa (Fig. 3, right panels). ATRX coprecipitated with Daxx, but ATRX deletion did not influence binding of either histone chaperone, as expected from our earlier studies (Lewis et al. 2010). ASF1 was constitutively associated with H3.3-HA.

We therefore conclude that the HIRA and Daxx complexes independently interact with H3.3 and likely maintain distinct pools of $\mathrm{H} 3.3 / \mathrm{H} 4$ units in the nucleus. This is in line with our recent observation that HIRA and ATRX/Daxx deposit histones at distinct genomic locations: HIRA at genic regions (Goldberg et al. 2010) and ATRX/Daxx at telomeres as well as pericentromeric heterochromatin (Drané et al. 2010; Lewis et al. 2010).

Although our in vitro studies confirmed that Daxx directly interacts with the H3.3 AAIG motif (Lewis et al. 2010), similar direct evidence is thus far missing for HIRA. Together with previous data, our finding that HIRA consistently copurifies with $\mathrm{H} 3.3$ in a complex with Cabin-1 and Ubinuclein-1 (Tagami et al. 2004; Lewis et al. 2010) raises the possibility that these components contribute to binding and specificity as well. 


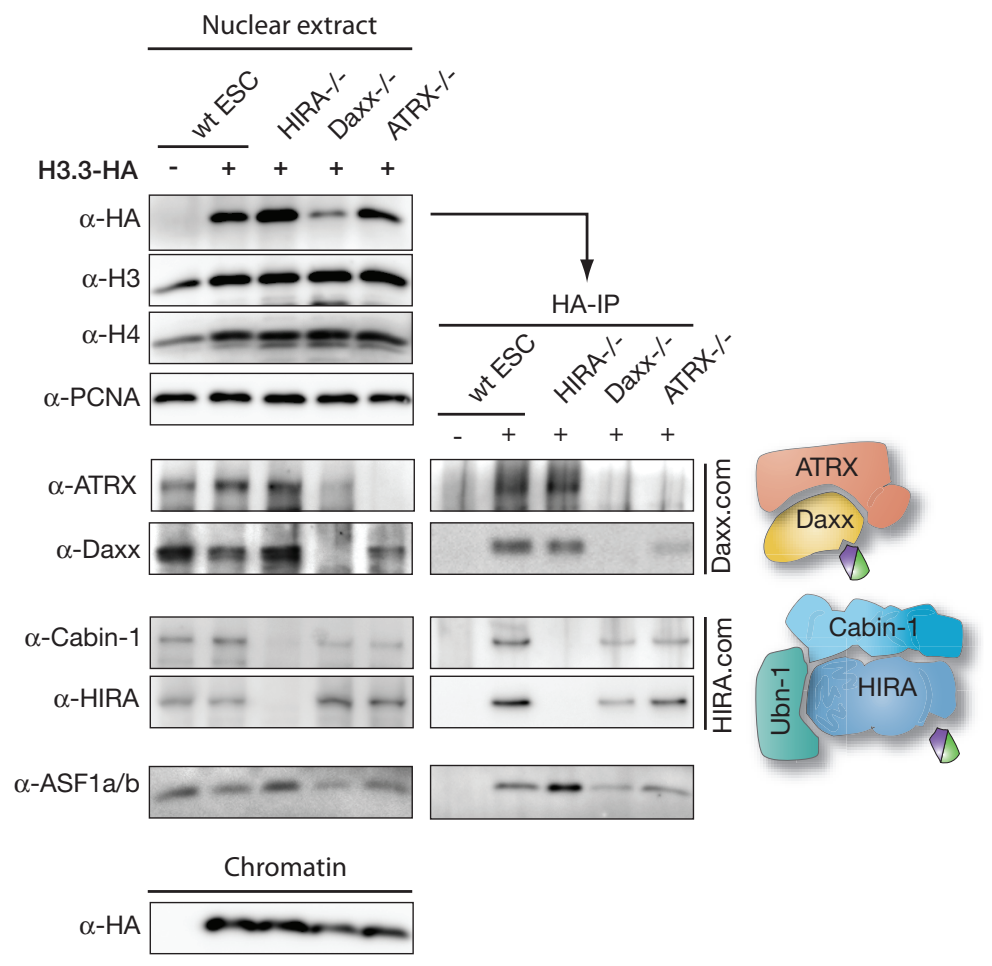

Figure 3. HIRA and Daxx independently associate with nucleoplasmic H3.3. Nuclear extracts were prepared from wild-type, $\mathrm{HIRA}^{+-}, \mathrm{Daxx}^{-1}$, and ATRX ${ }^{-1}$ mouse ESC lines (Roberts et al. 2002; Lewis et al. 2010). Where indicated, one $\mathrm{H} 3.3$ allele has been endogenously tagged with an H3.3B-HA knockin. Protein levels in the nuclear extracts and insoluble chromatin were assessed by immunoblotting (left panels). e-H3.3 was immunoprecipitated with $\alpha$-HA-agarose and eluted with $1 \%$ SDS. Copurifying proteins were analyzed by western blotting (right panels).

\section{DO PREDEPOSITION COMPLEXES REPRESENT INDEPENDENT HISTONE POOLS?}

Although H3.3 was originally characterized to be associated with actively transcribed genes (Ahmad and Henikoff 2002), the discovery of new H3.3-chaperone pathways and heterochromatic target regions (Hake et al. 2005; van der Heijden et al. 2007; Drané et al. 2010; Lewis et al. 2010; Santenard et al. 2010) poses some intriguing questions: Does H3.3 perform unique functions at distinct genomic locations? How can a single replacement variant contribute to gene activation or repression, depending on the chromatin context? How do the specialized deposition machineries contribute to the functional outcome of $\mathrm{H} 3.3$ incorporation?

The functional diversity of $\mathrm{H} 3.3$ could be explained if the distinct predeposition complexes would represent independent pools of $\mathrm{H} 3.3 / \mathrm{H} 4$ units. Assuming that the exchange between those pools is slow due to the biochemical stability of the complexes or their distinct spatial or temporal distribution during the cell cycle, the associated histones could acquire specific posttranslational modifications (PTMs) that might prime them for their respective functions at the genomic target sites.

Histone variants have been found enriched in PTMs that correlate with the local chromatin environment at their target sites (Hake et al. 2006). A set of specific modifications has been identified for the soluble pool of histones composed of newly synthesized histones but possibly also evicted histones "recycled" from chromatin: ubiquitous H4K5/K12 diacetylation (H4K5/K12diAc) (Sobel et al. 1995) and H3K9 monomethylation (H3K9me1) on both $\mathrm{H} 3.1 / 2$ and $\mathrm{H} 3.3$, as well as $\mathrm{H} 3 \mathrm{~K} 9$ dimethylation (H3K9me2) and $\mathrm{H} 3 \mathrm{~K} 9$ acety- lation (H3K9Ac) enriched on H3.3 (Loyola et al. 2006). Given the overall heterogeneity of predeposition PTMs on the single $\mathrm{H} 3.3 \mathrm{~K} 9$ residue, we wondered if any of these marks might be characteristic of a subset of predeposition H3.3 associated with a specific chaperone complex.

\section{Distinct Histone Posttranslational Modifications Are Present in Predeposition Complexes}

Using heparin fractionation to separate e-H3.3-containing complexes from HeLa cells, we identified a subpopulation of e-H3.3 that was enriched in H3K9Ac (Fig. 4A), a modification that is thought to be established on non-nucleosomal $\mathrm{H} 3$ by the histone acetyltransferase Gcn5 (Adkins et al. 2007). Because the enrichment of H3K9Ac tracked well with the HIRA complex, we further analyzed the PTMs present on histones within the CAF-1, HIRA, and Daxx complexes (Fig. 4B). We found that H3K9Ac on e-H3.3 associated with the HIRA complexes but was undetectable in the Daxx complex. Both complexes contained low levels of acetylated H4. e-H3.1 in the CAF-1 complex was enriched in $\mathrm{H} 4$ acetylation, with $\mathrm{H} 3 \mathrm{~K} 9 \mathrm{Ac}$ present at a lower level than on e-H3.3. With the differential H3K9Ac as a first indication, we conclude that unique $\mathrm{H} 3.3 / \mathrm{H} 4$ PTMs can be associated with predeposition complexes.

\section{A MODEL FOR THE DIFFERENT PATHWAYS OF H3.3 CHROMATIN ASSEMBLY}

Our biochemical characterization of the distinct H3.3 deposition machineries allows us to propose a model for the journey of histones from synthesis to incorporation at specific genomic location. Newly synthesized histones in 
A

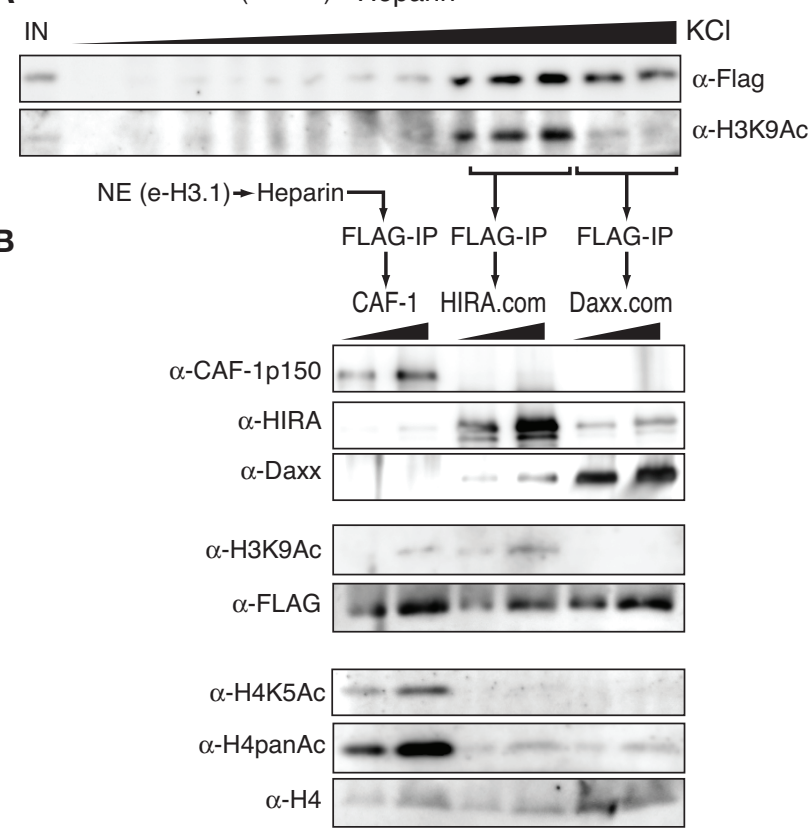

Figure 4. H3.3 predeposition complexes are associated with distinct histone posttranslational modifications. $(A)$ Western blot analysis of e-H3.3 lysine 9 acetylation (K9Ac) in the heparin salt gradient. K9Ac on e-H3.3 is enriched in fractions that correspond to the HIRA complex. $(B)$ The CAF-1, HIRA, and Daxx complexes were analyzed for selected $\mathrm{H} 3$ and $\mathrm{H} 4$ posttranslational modifications. K9Ac is enriched on e-H3.3 within the HIRA complex but is undetectable in the Daxx complex. the cytosol are likely immediately chaperoned by abundant general histone chaperones, such as ASF1 and/or NASP (Fig. 5, top). Furthermore, H4 is diacetylated on K5 and K12. ASF1-bound H3.3/H4 dimers then serve as substrates for various assembly pathways. Although a Daxx-H3.3/H4 complex has been found in the cytosol (Drané et al. 2010), HIRA seems to be exclusively localized to the nucleus and has not been found in a cytosolic complex with H3.3/H4 (Lorain et al. 1998; Tagami et al. 2004). ASF1 is ubiquitously detectable with H3.3/H4 (Tagami et al. 2004; Drané et al. 2010), but our biochemical data suggest that it is not a required component of HIRA and Daxx predeposition complexes (Fig. 2C,D) (Lewis et al. 2010).

\section{HIRA Complex and Histone Deposition at Transcribed Gene Bodies and Promoters}

HIRA histone chaperone activity has been well studied in Xenopus egg extracts, where it is necessary and sufficient for replication-independent chromatin assembly on exogenous DNA (Ray-Gallet et al. 2002, 2007; Tagami et al. 2004). However, in vivo, it accounts for only a subset of replication-independent chromatin assembly (Fig. 5, pathway 1), namely, within transcribed regions, at promoters and some regulatory elements (REs) (Goldberg et al. 2010). Evidence from yeast showing that the Hir complex and Asflp genetically and physically interact with the Set2 methyltransferase suggests that histone deposition might be functionally linked to H3K36 trimethylation. Subsequently, histone deacetylases remove acetylation marks from nucleosomes after RNA Pol passage.

H3.3 enrichment in gene bodies was consistently lost in HIRA $^{-1-}$ ESCs, but gene-expression patterns and the transcription elongation mark H3K36me3 were only mildly affected (Goldberg et al. 2010). Several other histone chaperones including Spt6 and FACT have also been implicated in histone exchange during transcription. Thus, the purpose of the transcription-associated H3.3 deposition and possible compensatory mechanisms in the absence of HIRA have yet to be defined.

The homology of HIRA to the yeast Hir corepressor complex subunits Hir1/2p suggests that its histone deposition activity might suppress basal transcription (Spector et al. 1997; Anderson et al. 2009). Furthermore, it will be necessary to define the roles of the remaining complex members Cabin-1 and Ubinuclein-1. Cabin-1 has recently been shown to act as a corepressor at p53 target genes, and it will be interesting to see if this activity requires HIRA (Jang et al. 2009). Ubinuclein-1 cooperates with HIRA in a chromatin assembly pathway specific to establishment of facultative heterochromatin domains in senescent cells (Fig. 5, pathway 2) (Banumathy et al. 2009).

\section{Daxx and ATRX Assemble H3.3 Chromatin at Pericentric Heterochromatin and Telomeres}

A considerable fraction of $\mathrm{H} 3.3$ is localized to telomeres in mouse ESCs, and we have recently shown that this enrichment is dependent on the Daxx/ATRX complex but not HIRA (Goldberg et al. 2010; Lewis et al. 2010). Whereas Daxx readily assembles H3.3/H4 chromatin in vitro, ATRX is required in vivo to target Daxx and $\mathrm{H} 3.3 / \mathrm{H} 4$ to the telomeres (Lewis et al. 2010) as well as to pericentric heterochromatin (Drané et al. 2010). Therefore, a diffusible Daxx-H3.3/H4 complex likely delivers histones to an ATRX-containing complex associated with telomeric chromatin (Fig. 5, pathway 3) (Wong et al. 2010). Furthermore, the chromatin-bound complex might contain the histone chaperone Dek and histone deacetylase HDACII, as suggested by biochemical studies (Hollenbach et al. 2002), to 


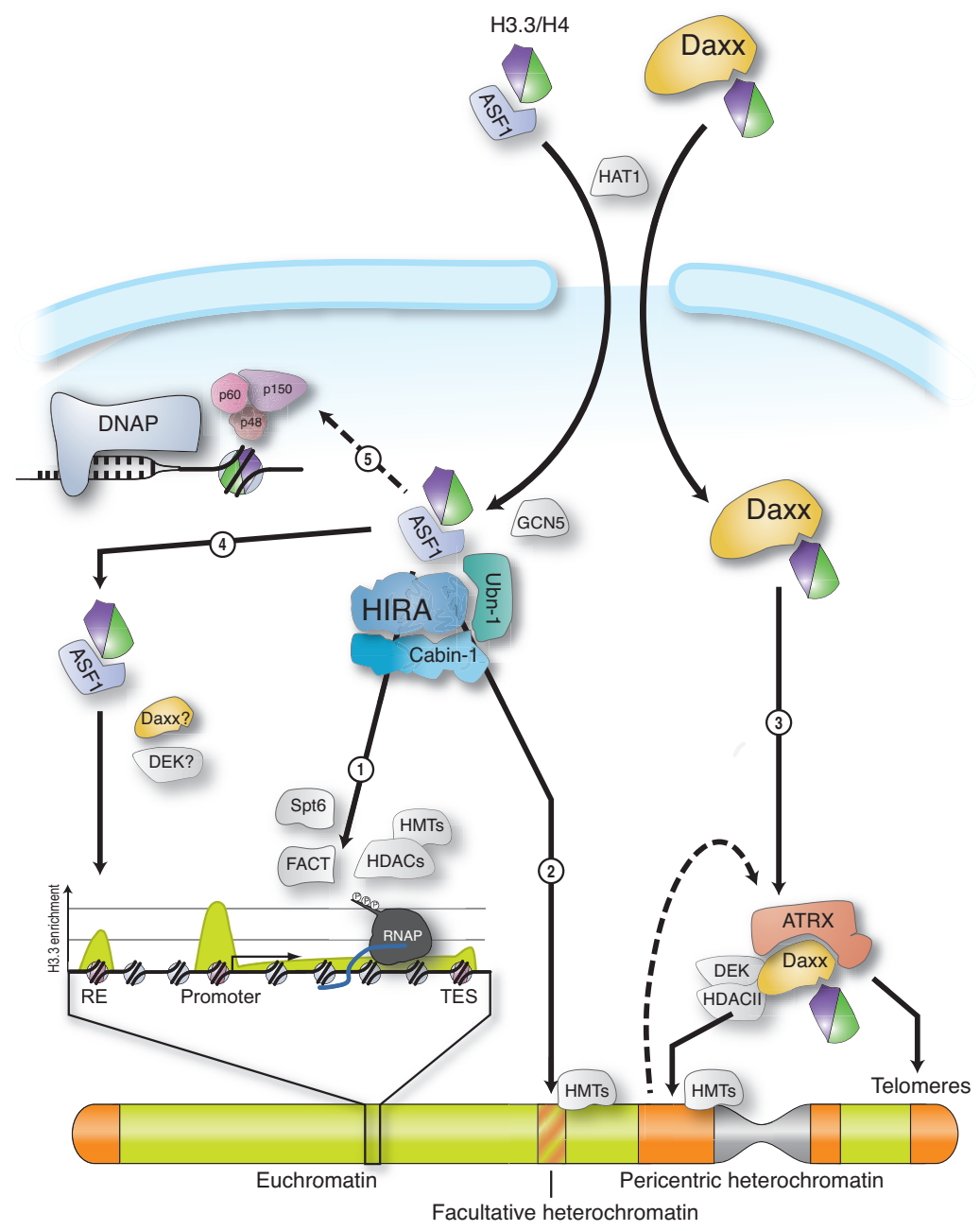

Figure 5. Predeposition complexes mediate H3.3 chromatin assembly. A model for the flow of histones from their synthesis in the cytosol (top) to their target genomic regions in the nucleus (bottom). Newly synthesized histones H3.3/H4 are chaperoned by ASF1 and a cytosolic pool of Daxx. HAT1 acetylates H4K5/K12 in the cytosol. Predeposition complexes HIRA.com and Daxx.com chaperone distinct pools of H3.3/H4. (1) HIRA.com mediates deposition of H3.3 at promoters and transcribed regions together with RNA polymerase (RNAP)-associated FACT (Spt16/SSRP1) and Spt6 histone chaperones. (2) HIRA.com deposits histones at facultative heterochromatin in senescent cells. Histone methyltransferases (HMTs) establish a repressive chromatin state. (3) Daxx.com cooperates with ATRX and possibly other factors in incorporating H3.3 into pericentric heterochromatin and telomeres. The chromatin-bound deposition complex might also contain the histone chaperone Dek and the histone deacetylase HDACII (4). An unknown factor delivers H3.3 to regulatory elements (REs). (5) In the absence of Daxx.com or HIRA.com, H3.3 associates with the replication-dependent CAF-1 complex that mainly deposits histones after the DNA polymerase (DNAP) replication fork.

prime a repressive chromatin state. This notion is supported by our finding that H3.3 does not carry the activating K9Ac mark in the Daxx complex (Fig. 4B).

Notably, Daxx is a highly conserved histone chaperone that appeared with the emergence of metazoans. Therefore, unlike the H3.3-chaperone activity of HIRA that is shared in yeast, Daxx likely evolved to diversify the functionality of H3.3 within the more complex chromatin structure of higher eukaryotes. A specific example is the asymmetric incorporation of histone $\mathrm{H} 3.3$ between the male and female pronucleus in the fertilized egg (Loppin et al. 2005; Torres-Padilla et al. 2006). Specific incorporation of $\mathrm{H} 3.3$ into the paternal genome is required for establishing heterochromatin in the decondensed sperm nucleus (Santenard et al. 2010). It is tempting to speculate that Daxx has taken on this and other metazoan-specific challenges.

\section{H3.3 Deposition at Regulatory Elements}

Many DNA sequences with regulatory functions including transcription factor-binding sites (TFBSs), insulator elements, and origins of replication are characterized by fast histone exchange dynamics. These epigenetic regulatory elements acquire newly synthesized histones and are constitutively enriched in H3.3 (Mito et al. 2007; Nakayama et al. 2007; Jin et al. 2009; Deal et al. 2010; Goldberg et al. 2010). Neither HIRA nor ATRX deletion significantly altered the global incorporation of H3.3 at these sites (Goldberg et al. 2010), suggesting that a yet unknown assembly 
pathway is used. Although general histone chaperones such as ASF1 or NASP could provide the histones for ATP-dependent chromatin remodelers that occupy these locations, it is important to note that this pathway is also specific to H3.3 (Goldberg et al. 2010). We therefore speculate that Daxx, Dek, or yet unknown factors mediate the deposition of H3.3 at regulatory elements (Fig. 5, pathway 4). Dek has recently been found to cooperate with ATP-dependent chromatin remodelers and to act as a coactivator for nuclear receptors (Sawatsubashi et al. 2010).

Intriguingly, H3K4 monomethylation is another hallmark of regulatory elements; it tracks well with exchange hotspots and H3.3 enrichment in genome-wide analyses (Deal et al. 2010; Goldberg et al. 2010). Which histone methyltransferase activity is responsible for establishing the $\mathrm{H} 3 \mathrm{~K} 4 \mathrm{me} 1$ mark remains elusive, but we speculate that methylation and histone exchange are functionally linked at these genomic regions.

\section{Alternate Pathways for H3.3 Deposition}

As is apparent from the imperfect discrimination that can be observed in pure in vitro assays with variant-specific chaperones (Drané et al. 2010; Lewis et al. 2010), only the interplay and competition of variant-specific histone chaperones can explain the exquisite specificity of H3.3 incorporation observed in vivo. Whereas in unperturbed systems, H3.1 and H3.3 associate exclusively with their cognate chaperone systems (CAF-1, HIRA, and Daxx, respectively), we did observe CAF-1 copurifying with e-H3.3 in the absence of Daxx and HIRA (Fig. 5, pathway 5) (Drané et al. 2010; Lewis et al. 2010). Furthermore, there is evidence that low levels of H3.3 are broadly incorporated into chromosomes by the replication-dependent machinery in Drosophila cells (Ahmad and Henikoff 2002; Schwartz and Ahmad 2005). Therefore, similar to the single yeast H3.3like histone, H3.3 might represent a universal variant for replication-dependent and -independent deposition pathways, i.e., simply outnumbered by the large pool of H3.1/2 present during $\mathrm{S}$ phase. Additionally, studies in flies suggest that H3.1/2 can take over some but not all functions of H3.3 (Hödl and Basler 2009; Sakai et al. 2009).

\section{CONCLUSION}

As illustrated by the replacement histone H3.3, rapidly emerging literature points to remarkable complexity for the use of relatively minor histone variants in punctuating epigenomes in organisms ranging from yeast to man. Multiple predeposition and assembly systems are called on to deposit histone variants in select genomic locations. Although these variants often differ in only a small number of amino acids, mounting evidence suggests that, once assembled, they perform specialized functions that remain poorly defined. It has been proposed that selective use of histone variants may contribute to a "nucleosome code" (Bernstein and Hake 2006), providing additional variation to that provided by chromatin remodeling and posttranslational modifications. We look forward to future experiments identifying and characterizing the predeposition complexes that engage these variants, giving the field more mechanistic insights into how these variants make their complicated journeys from synthesis to distinct chromatin functions.

\section{ACKNOWLEDGMENTS}

We thank Peter Scambler and David Pickett for cell lines and Peter Adams for monoclonal $\alpha$-HIRA antibody WC119. We also thank Peter Lewis for experimental guidance and intellectual input and Laura Banaszynski, Christian Zierhut, and Lindsey Baker for helpful discussions and critical reading of the manuscript. S.J.E was supported by a Boehringer Ingelheim Fonds fellowship; C.D.A acknowledges support from several National Institutes of Health grants and The Rockefeller University.

\section{REFERENCES}

Adkins MW, Tyler JK. 2004. The histone chaperone Asflp mediates global chromatin disassembly in vivo. J Biol Chem 279: 52069-52074.

Adkins MW, Carson JJ, English CM, Ramey CJ, Tyler JK. 2007. The histone chaperone anti-silencing function 1 stimulates the acetylation of newly synthesized histone $\mathrm{H} 3$ in S-phase. J Biol Chem 282: 1334-1340.

Ahmad K, Henikoff S. 2002. The histone variant H3.3 marks active chromatin by replication-independent nucleosome assembly. Mol Cell 9: 1191-1200.

Anderson HE, Wardle J, Korkut SV, Murton HE, Lopez-Maury L, Bahler J, Whitehall SK. 2009. The fission yeast HIRA histone chaperone is required for promoter silencing and the suppression of cryptic antisense transcripts. Mol Cell Biol 29: 51585167.

Andrews AJ, Chen X, Zevin A, Stargell LA, Luger K. 2010. The histone chaperone Nap1 promotes nucleosome assembly by eliminating nonnucleosomal histone DNA interactions. Mol Cell 37: 834-842.

Banumathy G, Somaiah N, Zhang R, Tang Y, Hoffmann J, Andrake M, Ceulemans H, Schultz D, Marmorstein R, Adams PD. 2009. Human UBN1 is an ortholog of yeast Hpc2p and has an essential role in the HIRA/ASF1a chromatin-remodeling pathway in senescent cells. Mol Cell Biol 29: 758-770.

Bernstein E, Hake SB. 2006. The nucleosome: A little variation goes a long way. Biochem Cell Biol 84: 505-517.

Campos EI, Reinberg D. 2010. New chaps in the histone chaperone arena. Genes Dev 24: 1334-1338.

Deal RB, Henikoff JG, Henikoff S. 2010. Genome-wide kinetics of nucleosome turnover determined by metabolic labeling of histones. Science 328: 1161-1164.

Drané P, Ouararhni K, Depaux A, Shuaib M, Hamiche A. 2010. The death-associated protein DAXX is a novel histone chaperone involved in the replication-independent deposition of $\mathrm{H} 3.3$ Genes Dev 24: 1253-1265.

Elsaesser SJ, Goldberg AD, Allis CD. 2010. New functions for an old variant: No substitute for histone H3.3. Curr Opin Genet Dev 20: 110-117.

Goldberg AD, Banaszynski LA, Noh K-M, Lewis PW, Elsaesser SJ, Stadler S, Dewell S, Law M, Guo X, Li X, et al. 2010. Distinct factors control histone variant $\mathrm{H} 3.3$ localization at specific genomic regions. Cell 140: 678-691.

Hake SB, Garcia BA, Kauer M, Baker SP, Shabanowitz J, Hunt DF, Allis CD. 2005. Serine 31 phosphorylation of histone variant H3.3 is specific to regions bordering centromeres in metaphase chromosomes. Proc Natl Acad Sci 102: 6344-6349.

Hake SB, Garcia BA, Duncan EM, Kauer M, Dellaire G, Shabanowitz J, Bazett-Jones DP, Allis CD, Hunt DF. 2006. Expression patterns and post-translational modifications associated with mammalian histone H3 variants. J Biol Chem 281: 559 568 . 
Hödl M, Basler K. 2009. Transcription in the absence of histone H3.3. Curr Biol 19: 1221-1226.

Hollenbach AD, McPherson CJ, Mientjes EJ, Iyengar R, Grosveld G. 2002. Daxx and histone deacetylase II associate with chromatin through an interaction with core histones and the chromatin-associated protein Dek. J Cell Sci 115: 3319-3330.

Jang H, Choi SY, Cho EJ, Youn HD. 2009. Cabin1 restrains p53 activity on chromatin. Nat Struct Mol Biol 16: 910-915.

Jin C, Zang C, Wei G, Cui K, Peng W, Zhao K, Felsenfeld G. 2009. H3.3/H2A.Z double variant-containing nucleosomes mark "nucleosome-free regions" of active promoters and other regulatory regions. Nat Genet 41: 941-945.

Lewis PW, Elsaesser SJ, Noh K-M, Stadler SC, Allis CD. 2010. Daxx is an H3.3-specific histone chaperone and cooperates with ATRX in replication-independent chromatin assembly at telomeres. Proc Natl Acad Sci 107: 14075-14080.

Loppin B, Bonnefoy E, Anselme C, Laurençon A, Karr TL, Couble P. 2005. The histone H3.3 chaperone HIRA is essential for chromatin assembly in the male pronucleus. Nature 437: 1386-1390.

Lorain S, Quivy JP, Monier-Gavelle F, Scamps C, Lécluse Y, Almouzni G, Lipinski M. 1998. Core histones and HIRIP3, a novel histone-binding protein, directly interact with WD repeat protein HIRA. Mol Cell Biol 18: 5546-5556.

Loyola A, Bonaldi T, Roche D, Imhof A, Almouzni G. 2006. PTMs on $\mathrm{H} 3$ variants before chromatin assembly potentiate their final epigenetic state. Mol Cell 24: 309-316.

Meshorer E, Yellajoshula D, George E, Scambler PJ, Brown DT, Misteli T. 2006. Hyperdynamic plasticity of chromatin proteins in pluripotent embryonic stem cells. Dev Cell 10: 105-116.

Mito Y, Henikoff JG, Henikoff S. 2007. Histone replacement marks the boundaries of cis-regulatory domains. Science 315: 1408-1411.

Nakayama T, Nishioka K, Dong YX, Shimojima T, Hirose S. 2007. Drosophila GAGA factor directs histone H3.3 replacement that prevents the heterochromatin spreading. Genes Dev 21: 552-561.

Ray-Gallet D, Quivy JP, Scamps C, Martini EM, Lipinski M, Almouzni G. 2002. HIRA is critical for a nucleosome assembly pathway independent of DNA synthesis. Mol Cell 9: 10911100.

Ray-Gallet D, Quivy JP, Silljé HW, Nigg EA, Almouzni G. 2007. The histone chaperone Asf1 is dispensable for direct de novo histone deposition in Xenopus egg extracts. Chromosoma 116: 487-496.

Roberts C, Sutherland HF, Farmer H, Kimber W, Halford S, Carey A, Brickman JM, Wynshaw-Boris A, Scambler PJ. 2002. Targeted mutagenesis of the Hira gene results in gastrulation de- fects and patterning abnormalities of mesoendodermal derivatives prior to early embryonic lethality. Mol Cell Biol 22: 23182328 .

Sakai A, Schwartz BE, Goldstein S, Ahmad K. 2009. Transcriptional and developmental functions of the $\mathrm{H} 3.3$ histone variant in Drosophila. Curr Biol 19: 1816-1820.

Santenard A, Ziegler-Birling C, Koch M, Tora L, Bannister AJ, Torres-Padilla M-E. 2010. Heterochromatin formation in the mouse embryo requires critical residues of the histone variant H3.3. Nat Cell Biol 12: 853-862.

Sawatsubashi S, Murata T, Lim J, Fujiki R, Ito S, Suzuki E, Tanabe M, Zhao Y, Kimura S, Fujiyama S, et al. 2010. A histone chaperone, DEK, transcriptionally coactivates a nuclear receptor. Genes Dev 24: 159-170.

Schwartz BE, Ahmad K. 2005. Transcriptional activation triggers deposition and removal of the histone variant H3.3. Genes Dev 19: 804-814.

Sharp JA, Franco AA, Osley MA, Kaufman PD. 2002. Chromatin assembly factor I and Hir proteins contribute to building functional kinetochores in S. cerevisiae. Genes Dev 16: 85-100.

Sobel RE, Cook RG, Perry CA, Annunziato AT, Allis CD. 1995. Conservation of deposition-related acetylation sites in newly synthesized histones H3 and H4. Proc Natl Acad Sci 92: 12371241.

Spector MS, Raff A, DeSilva H, Lee K, Osley MA. 1997. Hirlp and Hir $2 p$ function as transcriptional corepressors to regulate histone gene transcription in the Saccharomyces cerevisiae cell cycle. Mol Cell Biol 17: 545-552.

Tagami H, Ray-Gallet D, Almouzni G, Nakatani Y. 2004. Histone $\mathrm{H} 3.1$ and $\mathrm{H} 3.3$ complexes mediate nucleosome assembly pathways dependent or independent of DNA synthesis. Cell 116: 51-61.

Torres-Padilla M-E, Bannister AJ, Hurd PJ, Kouzarides T, Zernicka-Goetz M. 2006. Dynamic distribution of the replacement histone variant $\mathrm{H} 3.3$ in the mouse oocyte and preimplantation embryos. Int J Dev Biol 50: 455-461.

van der Heijden GW, Derijck AAHA, Pósfai E, Giele M, Pelczar P, Ramos L, Wansink DG, van der Vlag J, Peters AHFM, de Boer P. 2007. Chromosome-wide nucleosome replacement and H3.3 incorporation during mammalian meiotic sex chromosome inactivation. Nat Genet 39: 251-258.

Wong LH, McGhie JD, Sim M, Anderson MA, Ahn S, Hannan RD, George AJ, Morgan KA, Mann JR, Choo KHA. 2010. ATRX interacts with H3.3 in maintaining telomere structural integrity in pluripotent embryonic stem cells. Genome Res 20: 351-360. 


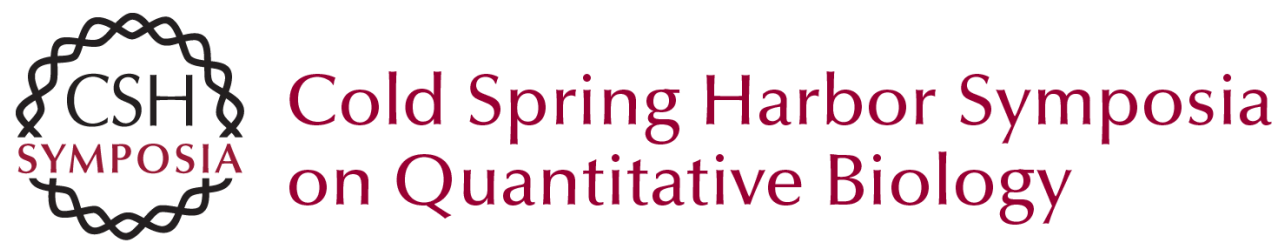

\title{
HIRA and Daxx Constitute Two Independent Histone H3.3-Containing Predeposition Complexes
}

\author{
S.J. Elsaesser and C.D. Allis
}

Cold Spring Harb Symp Quant Biol 2010 75: 27-34 originally published online November 3, 2010 Access the most recent version at doi:10.1101/sqb.2010.75.008

References This article cites 39 articles, 21 of which can be accessed free at: http://symposium.cshlp.org/content/75/27.full.html\#ref-list-1

\section{License}

Email Alerting Service top right corner of the article or click here. 